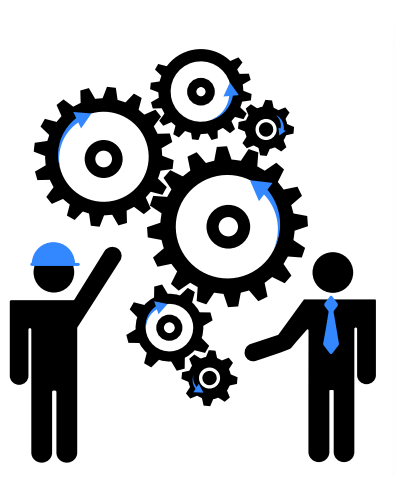

\title{
APLICACION DE LA SACAROSA COMO ADITIVO PARA CONTROLAR JUNTAS FRIAS EN EL CONCRETO
}

\author{
APPLICATION OF SUCROSE AS AN ADDITIVE TO CONTROL \\ COLD JOINTS IN CONCRETE
}

\author{
José Omar Cotrina Salvatierra ${ }^{1}$ \\ 1 Facultad de Ingeniería Civil, Universidad Continental. \\ E-mail:71782560@continental.edu.pe
}

\section{Resumen}

Esta investigación tiene como objetivo controlar las juntas frías en el concreto con aditivos específicos de sacarosa de $0,10 \%$ y o,40 \% como una alternativa para la construcción. La dosificación de azúcar puede ser una posibilidad real para los países en desarrollo, ya que está disponible en grandes cantidades y representa una fuente de consumo humano continua, además demostrar cómo esta adición de azúcar influye adecuadamente en las juntas frías en el concreto y no solo analizar su comportamiento, sino también de reducir costos en los proyectos de construcción.

Palabras clave: Juntas frías; concreto; sacarosa; asentamiento; fraguado.

\section{INTRODUCCIÓN}

Una de las funciones que cumplen los aditivos químicos en la tecnología del concreto es retardar la fragua, hecho que influye en la hidratación del cemento que tiene una lenta formación de los hidratos [1]. En las construcciones con concreto, de grandes extensiones de área, se originan diferencias en la resistencia por sectores de vaciado, a consecuencia de grandes intervalos de tiempo, por diferentes factores que originan las juntas frías.

La sacarosa en dosis de o,10 \% a o, 40\% permite controlar la aparición de juntas frías en concretos masivos. Tiene como propósito influir tanto en la minimización de costos como en la optimización del tiempo a selección del aditivo y el contenido de adición debe garantizar el efecto retardador durante

\begin{abstract}
This research aims to control the cold joints in the concrete with specific additives $0.10 \%$ and $0.40 \%$ sucrose as alternative for construction. The dosage of sugar can be a real possibility for developing countries since it is available in large quantities and represents a source of continuous human consumption, also demostrate how this addition of sugar properly influences the cold joints in the concrete in order not only to see its behavior but also to reduce costs in construction projects.
\end{abstract}

Keywords: cold joints; concrete; sucrose; settlement; setting.

el período inicial, favoreciendo el incremento de las propiedades mecánicas a edades avanzadas.

El objetivo principal es controlar las juntas frías con dosis de $0,10 \%$ a $0,40 \%$ de sacarosa en concretos masivos. La hipótesis está relacionada si al hacer uso de dosis de $0.10 \%$ y $0.40 \%$ la sacarosa disminuirá la aparición de juntas frías en concretos masivos y si el diseño de mezcla con la dosis de sacarosa no influirá en la resistencia final.

\section{RESULTADOS}

a) Caracterización de los agregados- Pilcomayo

Los agregados influyen en el concreto, tanto en el estado fresco como en el endurecido, y la efectividad en el procedimiento de control de calidad 
tiene su efecto en las variaciones de las propiedades del concreto [2]. Por tal motivo, las caracterizaciones de los agregados finos (arena) y gruesos (piedra) hasta el concreto fresco y/o endurecido se realizan según la Norma Técnica Peruana (en adelante NTP).

En la Tabla 1 se muestran los resultados de los agregados de la cantera de Pilcomayo (agregado fino y agregado grueso), realizados mediante ensayos de acuerdo con la NTP y la American Society for Testing Materials (ASTM). Para hacer el diseño de mezcla, se añadió o,10 \% y o,40 \% de sacarosa en el concreto; todas las dosis están en función al peso de una bolsa de cemento de 42,5 kg.

Las Figuras 1 y 2 muestran las curvas granulométricas de agregados fino y grueso que están dentro de los parámetros Husos C y 56, respectivamente, que son los límites permisibles.

Tabla 1. Caracterización de agregados

\begin{tabular}{|c|c|c|}
\hline & \multicolumn{2}{|c|}{ Caracterización de los agregados } \\
\hline & Ar & Pd \\
\hline P. específico & 2.55 & 2.61 \\
\hline Humedad & 3.73 & 0.4 \\
\hline Absorción & 2.25 & 1.42 \\
\hline PUS & 1723 & 1484 \\
\hline PUC & 1903 & 1680 \\
\hline MF & 2.84 & $7 \cdot 58$ \\
\hline Tnmax & & $3 / 4$ \\
\hline Tmax & & 1 \\
\hline Dnmax & & 1 \\
\hline
\end{tabular}

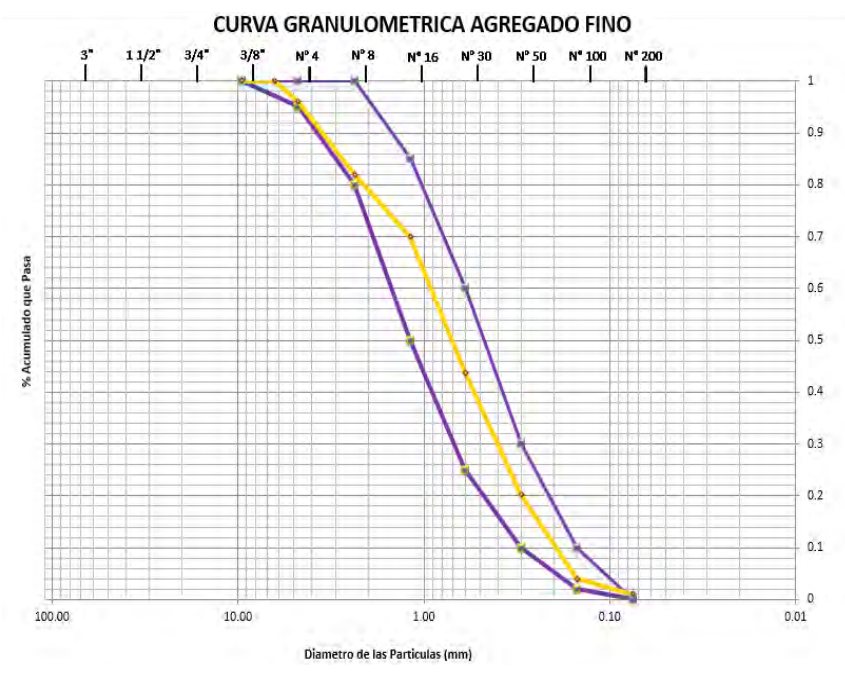

Figura 1. Granulométrica agregado fino - Huso C

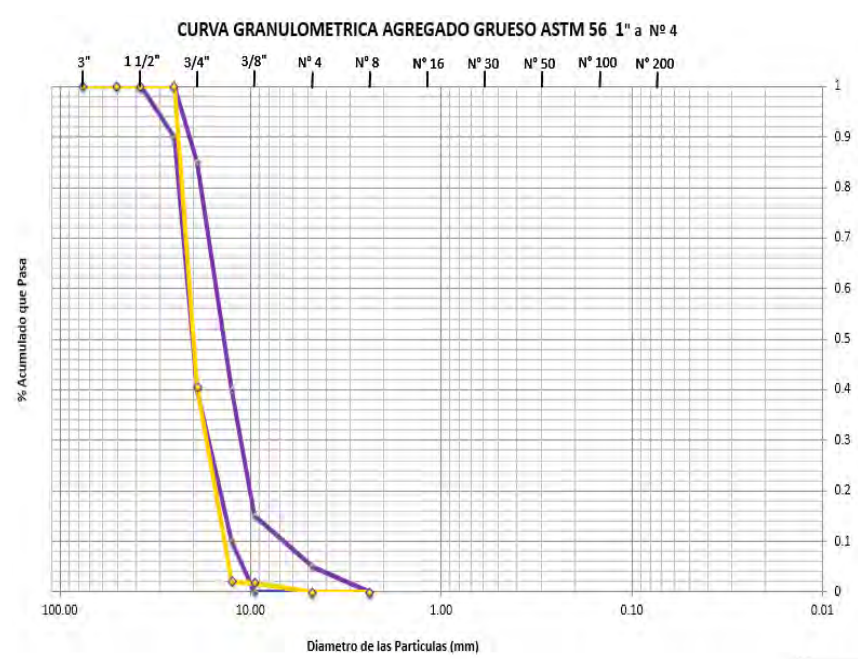

Figura 2. Granulometría agregado grueso - Huso 56

El módulo de finura de agregado fino en el concreto es resistente, del agregado grueso su finura es gruesa y en su peso específico es un agregado normal.

\section{b) Diseño de mezcla por el métodoglobal con aditivo}

El diseño de mezcla por el método global logra una óptima trabajabilidad y resistencia, así mismo se obtienen cantidades de cemento, agua, piedra, arena, \% aire para utilizar en proporción del cemento.

Tabla 2. Proporciones de acuerdo con la dosis

\begin{tabular}{|c|c|c|c|c|}
\hline & Descripción & $\begin{array}{c}\text { Dosis } \\
\mathbf{0 , 0 0} \%\end{array}$ & $\begin{array}{c}\text { Dosis } \\
\mathbf{0 , 4 0} \%\end{array}$ & $\begin{array}{c}\text { Dosis } \\
\mathbf{0 , 1 0} \%\end{array}$ \\
\hline $\mathbf{1}$ bolsa $(\mathbf{k g})$ & $\mathbf{1} / \mathbf{2}$ bolsa & $\mathbf{1} / \mathbf{2}$ bolsa & $\mathbf{1} / \mathbf{2}$ bolsa \\
\hline $\mathbf{C}$ & 42,5 & 21,25 & 21,25 & 21,25 \\
\hline $\mathbf{H 2 O}$ & 27,2 & 13,6 & 13,6 & 13,6 \\
\hline $\mathbf{P d}$ & 115,6 & 57,8 & 57,8 & 57,8 \\
\hline $\mathbf{A r}$ & 131,33 & 65,67 & 65,67 & 65,67 \\
\hline Dosis & & $0 \mathrm{~g}$ & $21,25 \mathrm{~g}$ & $85 \mathrm{~g}$ \\
\hline
\end{tabular}

\section{c) Propiedades de la sacarosa y su uso como aditivo}

Los aditivos pueden ser materiales orgánicos o inorgánicos. Un aditivo retardador trabaja sobre el aluminato tricálcico retrasando la reacción [3]. En los antecedentes de investigación se muestra que la sacarosa cumple como aditivo retardador, pero hasta cierta cantidad. 
En la Figura 3 se pueden observar las características y parámetros conceptuales a los que se puede aplicar un aditivo retardador. Se evidencia, además, que las acciones y funciones que cumple la sacarosa se asemejan a un aditivo de Tipo D, que es un reductor de agua y retardador. En la Tabla 3 se hace una comparación determinada de la sacarosa y un aditivo Sika Plastiment TM 12.

Tabla 3. Propiedades de la sacarosa y el aditivo químico Plastiment.

\begin{tabular}{|l|c|c|}
\hline Propiedad & Azúcar (sacarosa) & $\begin{array}{c}\text { Aditivo Sika } \\
\text { Plastiment TM } \mathbf{1 2}\end{array}$ \\
\hline Estado físico & Sólido & Líquido \\
\hline Color & Rubio & Marrón claro \\
\hline Solubilidad & Soluble en agua & Miscible \\
\hline $\mathrm{Ph}$ & $8 \mathrm{a} 8,5$ & $9+/-1,0$ \\
\hline Densidad & $1.59 \mathrm{~g} / \mathrm{cm} 3$ & $1,17+/-0,02 \mathrm{~g} / \mathrm{cm} 3$ \\
\hline
\end{tabular}

Fuente: http://www.complejocartavio.com.pe/productos_ AzuRubiaDomestica.html

\section{Plastiment ${ }^{\approx}$ TM 12}

\section{Retardante de Fragua Reductor de Agua}

\begin{tabular}{ll}
$\begin{array}{l}\text { Descripcion } \\
\text { General }\end{array}$ & Aditivo plastificante y retardante de fragua, exento de cloruros. \\
\hline Campos de aplicación & Vaciado de concreto en tiempo caluroso. \\
& Vaciado de concreto en grandes volumenes. \\
& Evita juntas frias en faenas contrinas. \\
& Concreto premezclado. \\
& Transporte de concrelo a largas distancas. \\
& Concreto bombeado.
\end{tabular}

Figura 3. Propiedades del aditivo reductor de agua y retardador

\section{d) Composición físico-química del azúcar o sa- carosa rubia $\left(\mathrm{C}_{12 \mathrm{H}_{22} \mathrm{OH}}\right)$}

Es el producto sólido cristalizado obtenido directamente del jugo de la caña de azúcar (Saccharum), mediante procedimientos apropiados; está constituido esencialmente por cristales de sacarosa.

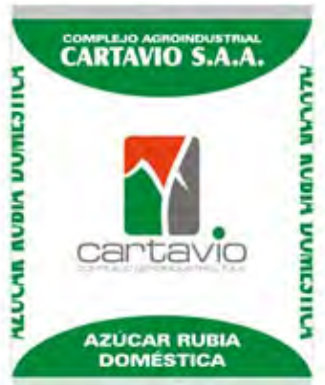

Figura 4. Empresa Complejo agroindustrial CARTAVIO S. A. A.
En la Tabla 4 se muestran las especificaciones técnicas de la sacarosa para trabajar con enlaces químicos en el concreto con el aluminato tricálcico, para retrasar la acción y así hacer el control de las juntas frías en paños de concreto, y evitar las fisuras con la adición de sacarosa en dosis del o,10 \% y o,40 \%. Sin embargo, según [2], hay que tener cuidado con el exceso de las sobredosificaciones ya que podría bajar la resistencia del concreto. Por lo tanto, el uso de la sacarosa en cantidades mayores puede también traer efectos negativos al concreto, por lo que se busca optar por una dosificación óptima.

Tabla 4. Características fisicoquímicas de la sacarosa

\begin{tabular}{|c|c|}
\hline $\begin{array}{l}\text { Características } \\
\text { fisicoquímicas }\end{array}$ & Especificaciones técnicas \\
\hline Polarización & $\begin{array}{c}\text { Mínimo de } 98,5 \% \text { / Máximo de } \\
99,60 \%\end{array}$ \\
\hline Humedad $(\% \mathrm{w} / \mathrm{w})$ & Máximo de o,4 \% \\
\hline Cenizas $(\% \mathrm{w} / \mathrm{w})$ & $\begin{array}{l}\text { Menor o igual a o,40 w/w (por } \\
\text { conductividad) }\end{array}$ \\
\hline Azúcar invertido (\% w/w) & Máximo de o,35\% \\
\hline Color & $\begin{array}{l}\text { Menor de } 1500 \text { unidades } \\
\text { ICUMSA }\end{array}$ \\
\hline Sedimentos & No mayor a $400 \mathrm{mg} / \mathrm{kg}$ \\
\hline $\begin{array}{l}\text { Características } \\
\text { microbiológicas }\end{array}$ & Requisitos \\
\hline $\begin{array}{l}\text { Microorganismos aero- } \\
\text { bios mesófilos viables }\end{array}$ & Menor de $100 \mathrm{ufc} / 10 \mathrm{~g}$ \\
\hline $\begin{array}{l}\text { Características } \\
\text { Sensoriales }\end{array}$ & Requisitos \\
\hline Apariencia & $\begin{array}{l}\text { Ausencia de cuerpos extraños } \\
\text { fácilmente detectables }\end{array}$ \\
\hline Otras características & Requisitos \\
\hline Empaque & $\begin{array}{l}\text { Empaque aprobado para uso } \\
\text { alimenticio por las autoridades } \\
\text { nacionales de salud. }\end{array}$ \\
\hline Rotulado & $\begin{array}{l}\text { De acuerdo con lo establecido en } \\
\text { el Decreto Supremo N. } .^{\circ} \text { o07-98- } \\
\text { SAya la Leyde Rotulado N. }{ }^{\circ} 28405\end{array}$ \\
\hline Almacenamiento & $\begin{array}{l}\text { Almacenado según normas legal- } \\
\text { es Decreto Supremo oo7-98-SA } \\
\text { Artículo } 72^{\circ} \text {. Almacenamiento } \\
\text { bajo techo, sobre parihuelas lim- } \\
\text { pias y secas, y en almacenes que } \\
\text { permiten la circulación de aire. }\end{array}$ \\
\hline
\end{tabular}

Fuente: http://www.complejocartavio.com.pe/productos_ AzuRubiaDomestica.html 


\section{e) Granulometría de la sacarosa}

En la Figura 5 se presenta la curva de gradación de la sacarosa en dosis de o,10 \% y o,40 \% para ser diluida con el agua; en la Figura 6 se muestra cómo se desarrolló el tamizado del azúcar y en la Tabla 5 se observan los resultados obtenidos.

Tabla 5. Gradación de la sacarosa

\begin{tabular}{|c|c|c|c|c|}
\hline \multicolumn{5}{|c|}{ Gradación del azúcar rubia } \\
\hline Tamiz & Peso & \multicolumn{3}{c|}{$\%$} \\
\hline ASTM & Ret. & Ret. & Ret. & $\%$ \\
\hline 11/2» & & parcial & acum. & acum. \\
\hline 1» & & 0 & 0 & 100 \\
\hline 3/4» & & 0 & 0 & 100 \\
\hline 1/2» & & 0 & 0 & 100 \\
\hline 3/8» & & 0 & 0 & 100 \\
\hline 1/4» & & 0 & 0 & 100 \\
\hline № 4 & 0 & 0 & 0 & 100 \\
\hline № 8 & 0 & 0 & 0 & 100 \\
\hline № 16 & 36 & 7,2 & 7,2 & 92,8 \\
\hline № 30 & 381 & 76,2 & 83,4 & 16,6 \\
\hline № 50 & 75 & 15 & 98,4 & 1,6 \\
\hline № 100 & 3 & 0,6 & 99,0 & 1 \\
\hline № 200 & 0 & 0 & 99,0 & 1 \\
\hline F & 5 & 1 & 100 & 0 \\
\hline Total & 500 & & 2,88 & \\
\hline
\end{tabular}

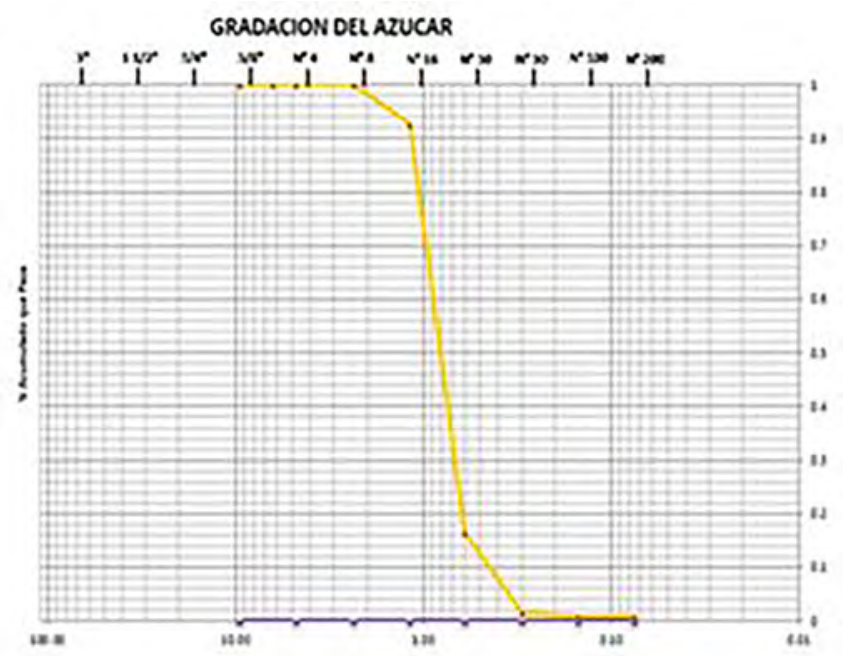

Figura 5. Curva granulométrica de la sacarosa

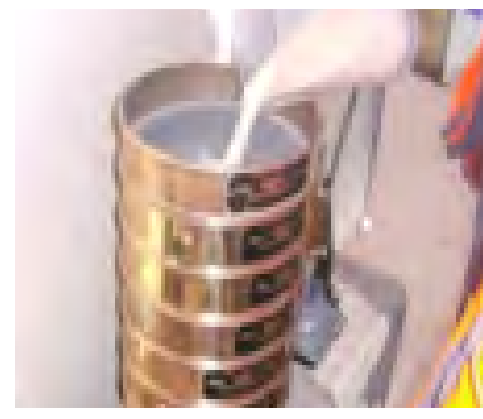

Figura 6. Tamizado del azúcar

f) Tiempo de fragua con dosificaciones de azú$\operatorname{car}(\mathbf{0}, 0 \%, 0,10 \%$ y $0,40 \%)$

En la Tabla 6 se muestran los tiempos del fraguado sin dosificación de azúcar: el fraguado inicial es de 2 horas con 10 minutos y el fraguado final, de 2 horas con 27 minutos.

Tabla 6. Tiempo de fragua inicial y final sin dosis

\begin{tabular}{|c|c|}
\hline \multicolumn{2}{|c|}{ Patrón (dosis o,oo \%) } \\
\hline TFI (min) & TFF (min) \\
\hline 130 & 147 \\
\hline
\end{tabular}

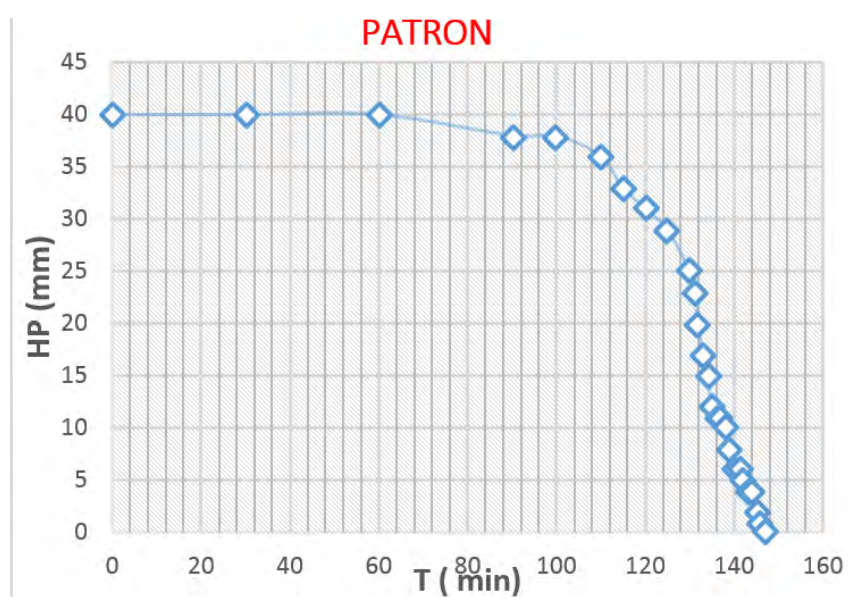

Figura 7. Tiempo de fragua sin dosis de azúcar o patrón

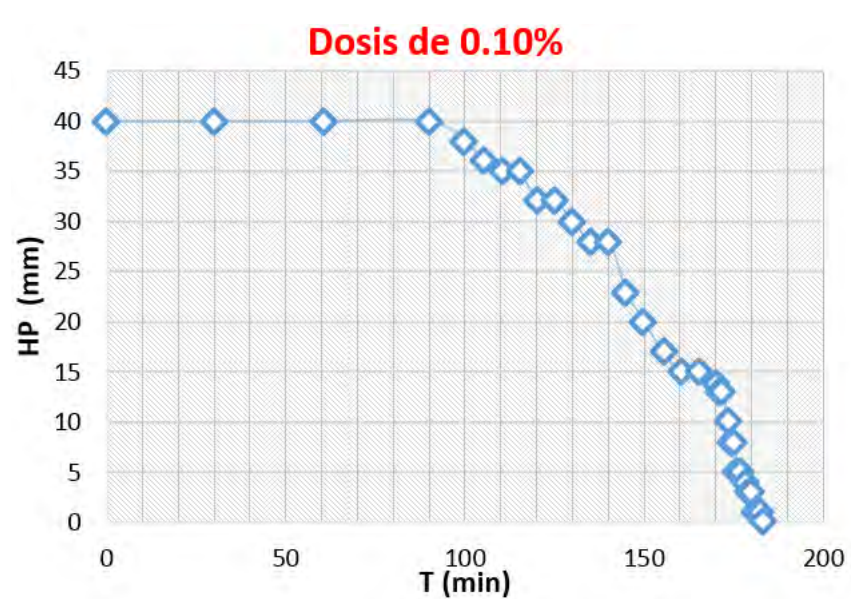

Figura 8. Tiempo de fragua con dosis de o,10 \% 
En la Tabla 7 se visualiza el tiempo de fraguado con dosis de o,10 \%; para el fraguado inicial fueron 2 horas con 23 minutos y el fraguado final se hizo en 3 horas con 3 minutos.

Tabla 7. Tiempo de fraguado con dosis de o,10 \%

\begin{tabular}{|c|c|}
\hline \multicolumn{2}{|c|}{ Patrón (dosis $\mathbf{0 . 1 0 \%}$ ) } \\
\hline $\begin{array}{c}\text { Tiempo de fragua inicial } \\
\text { (min) }\end{array}$ & $\begin{array}{c}\text { Tiempo de fragua final } \\
\text { (min) }\end{array}$ \\
\hline 143 & 183 \\
\hline
\end{tabular}

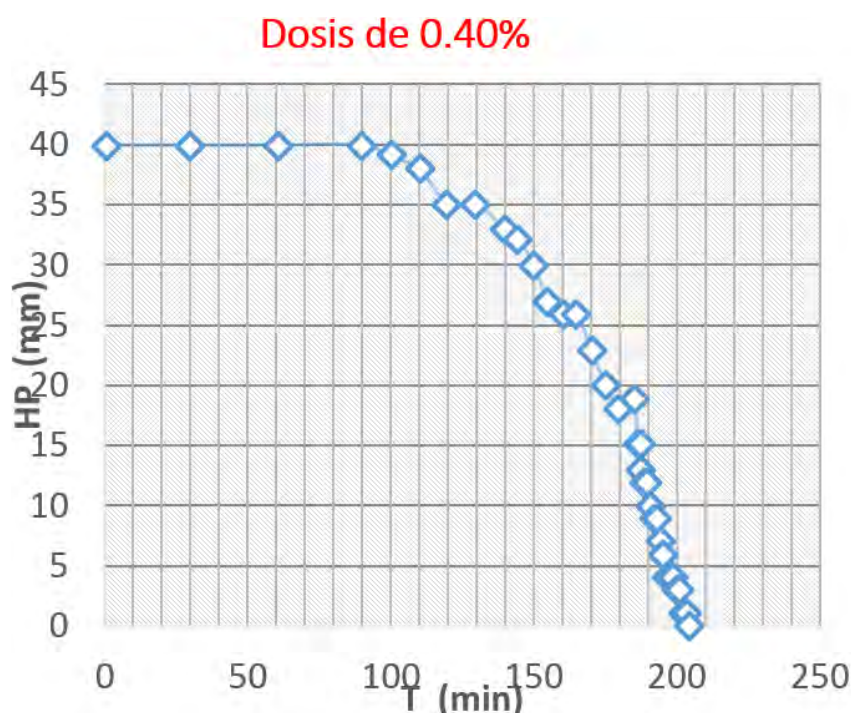

Figura 9. Tiempo de fragua con dosis de o,10 \%

En la Tabla 8, con una dosificación de $0.40 \%$, se obtiene un fraguado inicial de 2 horas con 47 minutos y un fraguado final de 3 horas con 24 minutos.

Tabla 8. Tiempo de fragua inicial (FI) y final (FF) con dosis de $0,40 \%$

\begin{tabular}{|c|c|}
\hline \multicolumn{2}{|c|}{ Patrón (dosis $\mathbf{0 , 4 0} \%)$} \\
\hline TFI (min) & TFF (min) \\
\hline 167 & 204 \\
\hline
\end{tabular}

Según [1], la muestra deberá ser empleada dentro de los 15 minutos siguientes, para que en este tiempo esté protegida ante cualquier acción de agentes atmosféricos. Estos ensayos deben ser de consistencia (asentamiento) y temperatura del concreto fresco.

\section{g) Asentamiento (slump)}

En la dosis de o \%, es decir sin sacarosa, tenemos un asentamiento de $5^{1 / 2}$ ».
El asentamiento de un concreto patrón, como se observa en la Figura 10, determina que el concreto tiene una consistencia plástica.

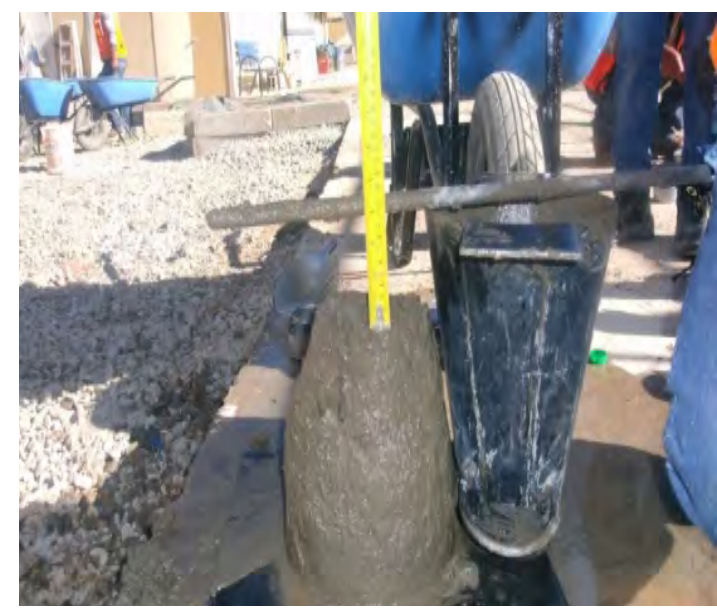

Figura 10. Asentamiento del concreto de $5^{1 / 2}$ »

Si la dosis de sacarosa en el concreto es de $0,10 \%$, se obtiene un asentamiento de 7 3/4».

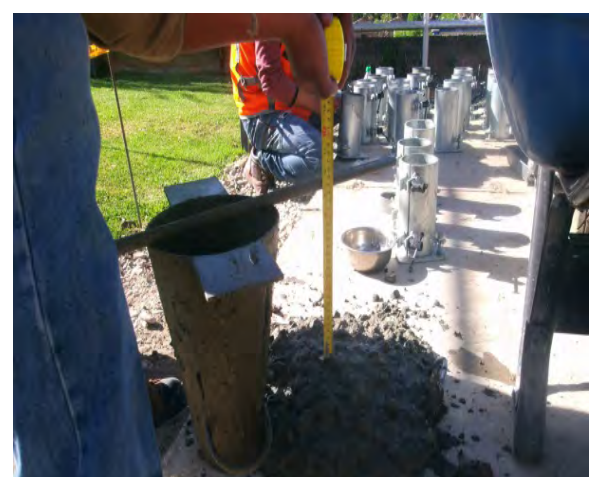

Figura 11. Asentamiento del concreto de $7^{1 / 2} \gg$

En el último ensayo, realizado con una dosis más alta de sacarosa (o,50 \%), se obtuvo un asentamien-

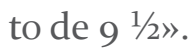

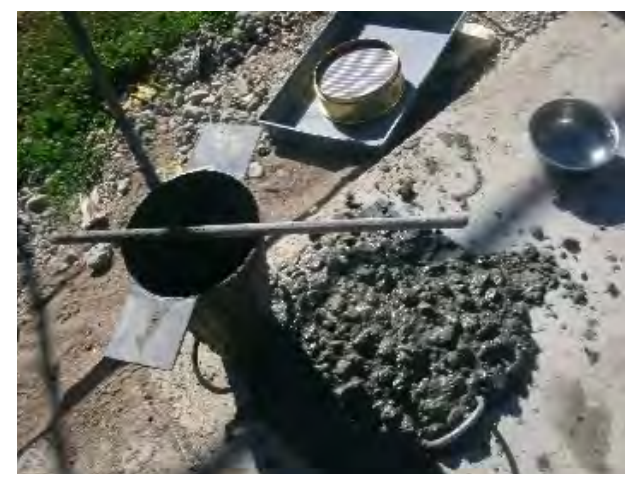

Figura 12. Asentamiento del concreto de $9^{1 / 2}$ »

Por lo tanto, el asentamiento de un concreto especial con sacarosa de o,40 \% determina que el concreto tiene una consistencia Rheo plástica. 
Los ensayos de asentamiento para clasificar al concreto por su consistencia fueron realizados según [4]. De los resultados obtenidos, podemos deducir lo siguiente:

- La determinación del asentamiento (slump) de 3 vaciados son de $0,10 \%, 0,40 \%$ de sacarosa y una sin dosis (modelo patrón), se especifica que es más trabajable (facilidad con que se desplaza el concreto) proporcionalmente cuando se añade sacarosa en la mezcla de concreto.

- La trabajabilidad de cada dosis de sacarosa determina la manera cómo su fluencia de la sacarosa a través de una reacción química hidrófila que reacciona la pasta con el agua que influye en la hidratación del cemento.

\section{h) Determinación de temperaturas}

La determinación de la temperatura se realizó de acuerdo a la NTP, especificada en [5].

Tabla 9. Temperaturas del agregado, agua y concreto

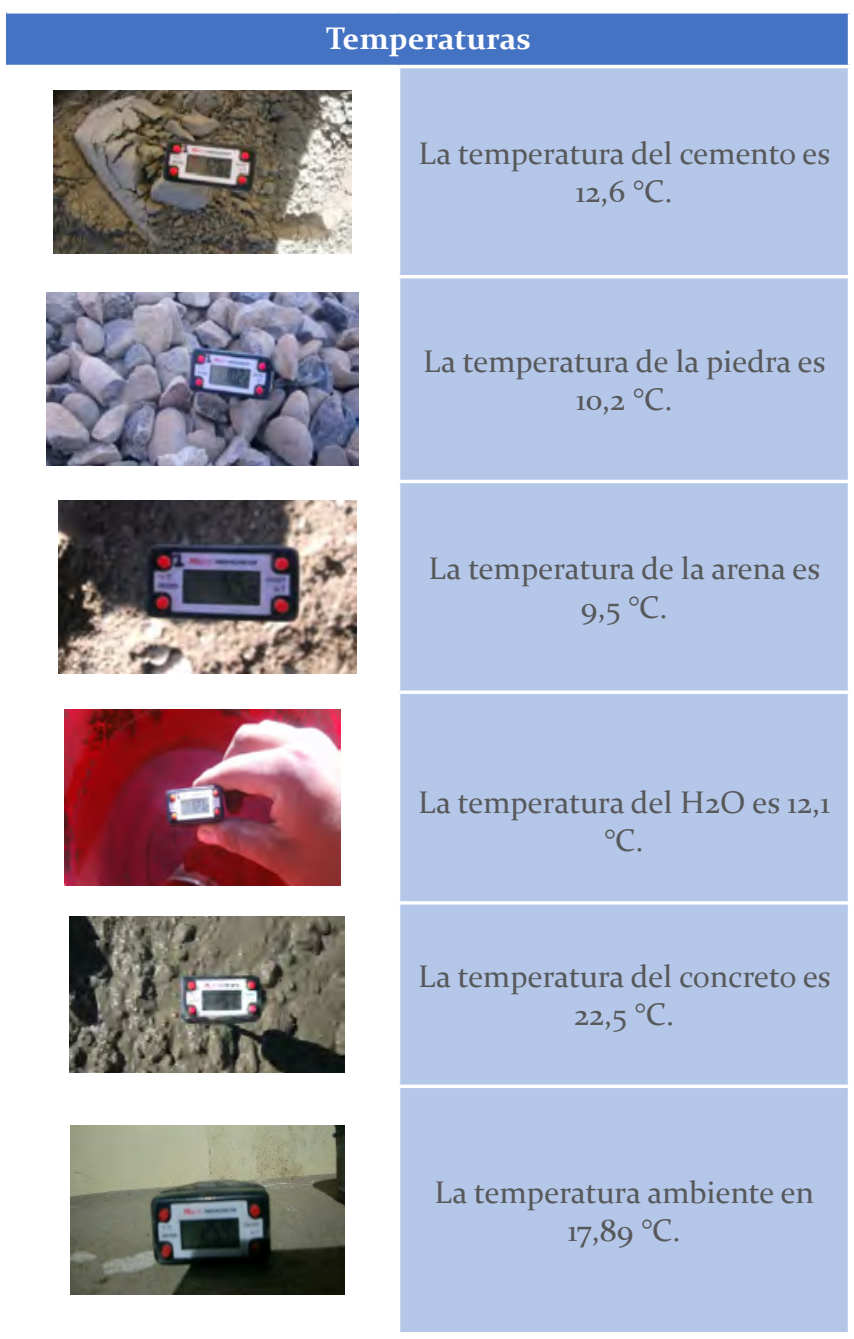

\section{DISCUSIÓN}

Con las características del agregado fino y grueso, se obtuvo el diseño de mezcla en la siguiente proporción:

$$
\begin{aligned}
& \text { C: } 1 \\
& \mathrm{H}_{2} \mathrm{O}: 0,64 \\
& \text { Pd: } 2,69 \\
& \text { Ar: 3,05 } \\
& \text { Aire: } 2 \%
\end{aligned}
$$

Con relación a la media bolsa de cemento en peso, tenemos :

$$
\begin{aligned}
& \mathrm{C}_{21.25} \mathrm{~kg} \\
& \mathrm{H}_{2} \mathrm{O} 14.03 \mathrm{~kg} \\
& \mathrm{Pd} 57,17 \mathrm{~kg} \\
& \mathrm{Ar} 65,03 \mathrm{~kg} \text { de sacarosa } \\
& 21,25 \mathrm{gr}(0,1 \%) \mathrm{y} \\
& 85 \mathrm{~g} \mathrm{(0,4 \% ).}
\end{aligned}
$$

La mezcla de concreto se realizó en tres tandas, los resultados se muestran en las Tablas 9 y 10.

Tabla 9. Resultados de las 3 dosis de asentamiento (Slump) y tiempo de fraguado

\begin{tabular}{|c|c|c|c|}
$\begin{array}{c}\text { Características } \\
\text { de concreto en } \\
\text { estado fresco }\end{array}$ & $\begin{array}{c}\text { M1 sin } \\
\text { azúcar }\end{array}$ & $\begin{array}{c}\text { M2 } \\
\text { con 21,25 g } \\
\text { de azúcar }\end{array}$ & $\begin{array}{c}\text { M3 } \\
\text { con 85 } \\
\% \text { de } \\
\text { azúcar }\end{array}$ \\
\hline Slump & $5^{1 / 2}$ & $7^{3 / 4}$ & $9^{1 / 2}$ \\
\hline Tfi (min.) & 130 & 143 & 167 \\
\hline Tff (min.) & 147 & 183 & 204 \\
\hline
\end{tabular}

Tabla 1o. Resultados de las roturas de 3 y 7 días

\begin{tabular}{c|c|c|c|}
\hline $\begin{array}{c}\text { Resistencia a } \\
\text { compresión }\end{array}$ & $\begin{array}{c}\text { M1 } \\
(\mathbf{0} \%)\end{array}$ & $\begin{array}{c}\text { M2 } \\
(\mathbf{0 , 1} \%)\end{array}$ & $\begin{array}{c}\text { M3 (0,4 } \\
\mathbf{\%})\end{array}$ \\
\hline 3 días & 88,91 & 99,60 & 1,63 \\
\hline 7 días & 137,37 & 106,07 & 7,64 \\
\hline
\end{tabular}

Al momento del vaciado, se observó que una mayor dosificación de azúcar produce (con respecto al patrón $\left.\mathrm{M}_{1}\right)$ :

\section{- Mayor trabajabilidad}

Dosis $0,1 \% \rightarrow 41 \%$

Dosis $0,4 \% \rightarrow 72 \%$ 


\section{- Mayor tiempo de fragua inicial}

Dosis $0,1 \% \rightarrow 10 \%$

Dosis $0,4 \% \rightarrow 29 \%$

\section{- Mayor tiempo de fragua final}

Dosis $0,1 \% \rightarrow 25 \%$

Dosis $0,4 \% \rightarrow 39 \%$

\section{CONCLUSIONES}

- La dosis de sacarosa al o,40 \% tiene un mejor tiempo de fragua en un $29 \%$ más que un concreto normal, pero tiene menos de $98 \%$ de resistencia.

- La dosis de sacarosa al o,10 \% tiene un $10 \%$ más de tiempo en fraguado final y una resistencia inicial de $12 \%$ más que un concreto normal, y es más admisible para juntas.

- Las dosis de sacarosa al o,10 \% y al o,40 \% son recomendables para grandes juntas frías y tienen una trabajabilidad de $41 \%$ y $72 \%$, respectivamente.

\section{RECOMENDACIONES}

- Se recomienda hacer ensayos con otras dosificaciones de sacarosa para buscar un resultado más óptimo en resistencia, consistencia y trabajabilidad respecto a su asentamiento.

- En las dosis de sacarosa al o,1 \% y al o,4\% se recomienda utilizar un aditivo incorporador de aire, debido a que durante el proceso de fraguado el concreto se encuentra expuesto a temperaturas menores a o ${ }^{\circ} \mathrm{C}$ (durante la noche).
- Según la roturación de los testigos, cuando se trabaja con el o,1 \% de azúcar, se recomienda desencofrar a los 3 días en una obra, ya que representa una resistencia al $12 \%$ mayor a la del concreto patrón.

\section{AGRADECIMIENTO}

$\mathrm{Al} \mathrm{Mg.} \mathrm{Ing.} \mathrm{Richard} \mathrm{Hugo} \mathrm{Reymundo} \mathrm{Gamarra,} \mathrm{por}$ sus enseñanzas y el conocimiento compartido que nos ha permitido aprender nuevas experiencias.

\section{REFERENCIAS}

[1] GAYOSO, R., GALVEZ, R., CUBA, M., Contribución al estudio de la sacarosa como aditivo retardador de la hidratación del cemento. España, 1993.

[2] RIVVA LÓPEZ, E. Supervisión del concreto en obra. Fondo Editorial del Instituto de la Construcción y Gerencia. Perú, 2004.

[3] PASQUEL CARBAJAL, E. Tópicos de Tecnología del concreto. Segunda edición. Lima: Colegio de Ingenieros del Perú, 1999.

[4] Norma Técnica Peruana: NTP 339.035 2009: Hormigón (concreto). Método de ensayo para la medición del asentamiento del concreto de cemento Portland INDECOPI N82C. Código N. ${ }^{\circ} 624.18341$

[5] NORMA TÉCNICA PERUANA (NTP) 339.184.2002: Método de ensayo normalizado para determinar la temperatura de mezclas del hormigón (concreto). Resolución 048-2008/CNB-INDECOPI. 